\title{
Top 10 research priorities for people living with pulmonary fibrosis, their caregivers, healthcare professionals and researchers
}

\author{
Gabriella Tikellis, ${ }^{1,2,3}$ Allison Tong, ${ }^{4}$ Joanna Y T Lee, ${ }^{1}$ Tamera J. Corte, ${ }^{3,4,5,6}$ \\ Alison J Hey-Cunningham, ${ }^{3,4}$ Michael Bartlett, ${ }_{1}^{7}$ Tonia Crawford, ${ }^{7}$ Ian Glaspole, ${ }^{1,3,8}$ \\ John Price, ${ }^{9}$ Jamie Maloney, ${ }^{7}$ Anne E Holland ${ }^{1,3,10}$
}

\begin{abstract}
- Additional material is published online only. To view please visit the journal online (http://dx.doi.org/10.1136/ thoraxjnl-2020-215731).

For numbered affiliations see end of article.
\end{abstract}

\section{Correspondence to} Dr Anne E Holland, Department of Physiotherapy, Alfred Health, Melbourne, VIC 3004, Australia; A.Holland@alfred.org.au

Received 4 July 2020

Revised 18 September 2020 Accepted 24 October 2020

Published Online First

4 December 2020

\section{GLinked}

http://dx.doi.org/10.1136/ thoraxjnl-2020-216616

Check for updates

(C) Author(s) (or their employer(s)) 2021. No commercial re-use. See rights and permissions. Published by BMJ.

To cite: Tikellis G, Tong A, Lee JYT, et al. Thorax

2021:76:575-581

\section{ABSTRACT}

Introduction People with pulmonary fibrosis (PF) experience a high symptom burden, reduced quality of life and a shortened lifespan. Treatment options are limited and little is known about what patients, caregivers and healthcare professionals (HCPs)/ researchers consider as the most important research priorities. This study aimed to identify the top 10 research priorities for PF across all stakeholders.

Methods Participants included people with PF, caregivers and HCPs/researchers involved with PF. The research priority setting exercise involved three stages: (1) identifying priorities using an open-ended questionnaire and thematic analysis, (2) development of specific research questions at a face-to-face workshop, and (3) online ranking of research questions to identify the top 10 research priorities using nominal group ranking method.

Results 196 participants completed stage 1 generating 560 questions and 14 research themes were identified. Stage 2 involved 32 participants and generated 53 indicative questions from which 39 were used for the final ranking. Stage 3 was completed by 270 participants. The top ranked priorities focussed on medications to reverse scarring in the lungs (ranked $1^{\text {st }}$ ), improving lung function (ranked $2^{\text {nd }}, 6^{\text {th }}$ and $8^{\text {th }}$ ), interventions aimed at alleviating symptoms (ranked $5^{\text {th }}$ and $7^{\text {th }}$ ), prevention of PF (ranked $3^{\text {rd }}$ and $4^{\text {th }}$ ) and the best exercise programme for PF (ranked $10^{\text {th }}$ ). There was good consensus among patients/carers and HCPs/researchers on the top 10 priorities, however, causes of acute exacerbations and early diagnosis for improving survival, was ranked higher by HCPs/researchers.

Conclusion Interventions for preserving lung health and alleviation of symptom burden were top research priorities for PF stakeholders.

\section{INTRODUCTION}

Pulmonary fibrosis (PF) is a group of life-limiting, interstitial lung diseases with no curative options and few effective treatments, despite decades of multidisciplinary research efforts. Idiopathic pulmonary fibrosis (IPF), PF of unknown aetiology, accounts for the majority of all cases, and is the form of PF that is most extensively researched. ${ }^{1}$ Recent research has confirmed that all forms of PF are generally progressive, leading to increasingly debilitating symptoms, falling quality of life, declining

\section{Key messages}

What is the key question?

- What are the most important research priorities for pulmonary fibrosis as determined by people with the disease, caregivers, healthcare professionals and researchers?

What is the bottom line?

- People with pulmonary fibrosis, caregivers and healthcare professionals/researchers collectively identified medications to reverse scarring in the lungs, medications to improve lung function and strategies to prevent pulmonary fibrosis as their top three most important priorities that should be addressed by research.

\section{Why read on?}

- This is the first research setting exercise for pulmonary fibrosis. The results will allow researchers, funders and policymakers to ensure that future research efforts are well aligned with stakeholder priorities.

lung function and ultimately shortened survival. ${ }^{2}$ To date, research efforts have mainly focussed on effective therapeutic options to reduce IPF's symptom burden and improve survival. However, the extent to which this is a shared priority of patients, caregivers and healthcare professionals (HCPs)/researchers has not been assessed in detail, such that there may be a mismatch between current research outputs and the priorities of people living with the disease. ${ }^{3}$

$\mathrm{PF}$ is associated with deterioration in lung function, dyspnoea, chronic cough and impaired quality of life. Patients often experience a relentless increase in symptoms that become distressing for themselves and caregivers and presents an ongoing challenge in maintaining quality of life. ${ }^{4}$ Proven treatments for PF are limited. Antifibrotic therapies (nintedanib and pirfenidone) slow the decline in lung function in people with IPF $^{5}$ and more recent findings suggest a similar role for nintedanib in people with other types of PF. ${ }^{2}$ However, while antifibrotic therapies slow down (rather than prevent) the decline in lung function, neither therapies have shown a beneficial effect on the debilitating symptoms and impaired 
quality of life. Therefore, most people with PF continue to experience a high symptom burden, a progressive decline in lung function and a reduced physical capacity that negatively impacts their physical, psychological and social well-being. ${ }^{6-8}$

As a life-limiting disease, there are many aspects of the PF journey that contribute to the dismal prognosis and psychological impact that require focussed research efforts. Yet, despite the gravity of the disease and its devastating impact on patients and families, there is a paucity of information regarding what research matters most to those living with the disease as well as those involved with their treatment and management. As such, current research priorities and research investments may not necessarily align with stakeholder priorities.

The last decade has seen a concerted effort to include patients and caregivers in establishing research priorities. Initiatives such as the UK-based James Lind Alliance (JLA) ${ }^{9}$ have developed frameworks for research priority setting projects that aim to raise the awareness of research questions that have direct relevance and potential benefits to patients, caregivers and HCPs. These initiatives bring together all stakeholders in a priority setting partnership with the objective of shaping research agendas by identifying and describing research priorities as determined by the stakeholders. Such partnerships have been developed for a range of conditions that include pulmonary hypertension, chronic kidney disease, cancer, diabetes and depression. ${ }^{10}$ However, no priority setting exercise has been undertaken for PF.

The aim of this study was to identify the research questions that are most important to people living with PF, their caregivers, HCPs and researchers, so as to better align stakeholder priorities to research efforts.

\section{METHODS}

A research priority setting exercise was undertaken based on the JLA framework for research priority setting partnership ${ }^{9}$ to produce a list of the 'top 10' research questions. Results were reported using the REporting guideline for PRIority SEtting of health research (REPRISE) guidelines. ${ }^{11}$ (online supplemental file 1)

\section{Eligibility}

Eligibility criteria for participation included (1) person with PF: diagnosed by a physician as having PF; (2) caregiver: identified as being involved in the care of someone with PF; (3) HCPs: involved in the diagnosis, treatment and/or management of people with PF or involved in research focussed on PF; (4) researchers: individuals involved in clinical or academic research focussing on PF. There were no exclusion criteria for this study and eligibility for participation in each stage was independent of participation in any previous stage.

This research priority setting exercise for PF involved three stages:

\section{Stage 1: Questionnaire - identifying important domains of research in $P F$}

Participants: People with PF and their caregivers were identified through the Australian Idiopathic Pulmonary Fibrosis Registry (AIPFR) and Lung Foundation Australia's (LFA) website and electronic direct mail (EDM) database. Healthcare professionals with experience in PF were identified through the Australian IPF Network (AUS IPF-net), a special interest group for HCPs also managed by LFA.
Methods: The initial questionnaire aimed to gather a wide range of experiences from people with $\mathrm{PF}$, their caregivers, HCPs and researchers. The questionnaire included one question with an open text response: 'What are the two most important problems for people with pulmonary fibrosis that you feel should be addressed by research?' Additional demographic information such as current age, gender, years living with PF and residential state/territory in Australia was also collected. The questionnaire was piloted by patients at an interstitial lung disease clinic to ensure clarity of items and developed as an online survey using Qualtrics XM software accessible through an anonymous link. Alternatively, a paper copy was posted if requested. The questionnaire remained open for 8 weeks from early July 2019 to end of August 2019. In addition, information at the end of the questionnaire provided respondents with an opportunity to register their interest in participating in stage 2 of the study, a face-toface workshop.

Consent: Implied consent was obtained through the completion of the questionnaire.

Analysis: Each of the 560 questions submitted as part of the stage 1 questionnaire were independently reviewed by two researchers (a senior qualitative researcher (AEH) and a midlevel researcher with experience in qualitative research (GT) using thematic analysis. This involved identifying 'key words' or codes in each response to identify patterns or themes. Duplicate or similar questions were incorporated under themes and no question was considered 'out of scope'. A total of 52 initial themes were identified that encompassed all 560 questions. The themes were then compared and discussed to generate a list of 14 overarching themes to guide discussion at the stage 2 workshop. The number of questions related to each of the 14 themes were tallied based on the proportion of total respondents to determine the order of the themes. Online supplemental file 2 lists the initial 52 themes, the number of questions that were categorised into each theme and how these related to the 14 overarching themes.

\section{Stage 2: Face-to-face workshop - development of specific research questions}

The objective of the face-to-face workshop was to use the themes generated in stage 1 to develop more specific research questions within each theme.

Participants: People with PF, caregivers, HCPs and researchers were invited to participate in stage 2 .

Methods: A half-day, face-to-face workshop was held in Melbourne, Australia, in August 2019. The workshop was promoted at the end of the stage 1 questionnaire as well as through LFA, inviting all stakeholders to be involved. The workshop involved small group discussions, each group led by a facilitator (AEH, JYTL, GT, and AJHC) with the overall process guided by an experienced facilitator (AT). During the workshop, the themes identified in stage 1 were presented to the collective group. The areas were discussed within each of the small groups with participants tasked with developing specific research questions based on the PICO model where possible: population $(\mathrm{P})$, intervention/indicator $(\mathrm{I})$, control $(\mathrm{C}$ - if applicable) and outcome $(\mathrm{O}) .{ }^{12}$ For example in the question 'What treatments are most effective in improving mental well-being (mood, anxiety, depression, optimism) in people with $P F$ ?' $\mathrm{P}=$ people with $\mathrm{PF}, \mathrm{I}=$ treatments and $\mathrm{O}=$ mental well-being including mood, anxiety, depression and optimism. Questions were documented on paper by a spokesperson from each group. Discussions at each table were audio recorded (with consent) to 
allow for the context of questions to be re-examined after the workshop.

Consent: Informed written consent was obtained in person at the workshop.

Analysis: In the last stage of the workshop, each participant within each group used coloured adhesive dots to indicate what for them, were the three most important questions from the group generated lists. The leading facilitator (AT) then collated the list of questions ranked by each group, to determine which questions were ranked as the top priorities and reported them back to the group.

\section{Stage 3: Prioritisation questionnaire - ranking of the top 10 research} priorities

Participants: The questionnaire was distributed to all stakeholders using a similar approach adopted in stage 1 .

Consent: Implied consent was obtained through the completion of the questionnaire.

Methods: The specific research questions generated during stage 2 were collated and reviewed by three researchers (GT, $\mathrm{AEH}$ and AT) to ensure that the content of the questions did not overlap, the wording was unambiguous and where possible, the question followed the PICO model. The final list of questions was developed into an online questionnaire using Qualtrics XM software, accessible through an anonymous link or made available in paper form if requested. The questionnaire was piloted with several IPF patients. Participants were asked to read through the list of questions and then identify the 10 questions that were most important to them by numbering them from 1 (most important) to 10 (least important).

A concerted effort was made to achieve a representative number of respondents across all stakeholder groups in particular, HCPs and researchers, by promoting the questionnaire through the Thoracic Society of Australia and New Zealand (TSANZ) weekly e-e-newsletter and local branch meetings and a more direct distribution of the questionnaire to colleagues in departments of respiratory medicine across local hospitals. The questionnaire remained open for 5 months from November 2019 to March 2020

Analysis: Demographic data was analysed descriptively as $\mathrm{n}$ (\% of total). Identification of the final 10 research priorities was based on the Nominal Group Technique used for ranking healthcare priorities. ${ }^{13}$ This involves computing an importance score for each question defined as the average of the reciprocal rankings. The reciprocal rank is defined as 1 over the rank assigned by the participant for that question. Questions that were not ranked by participants were given a 0 as the reciprocal ranking. The importance score ranges from 0 to 1 , with a higher score indicating a higher priority. Values approaching 1 indicate a highly prioritised outcome based on higher ranks and more frequent nominations, whereas values approaching 0 indicate infrequently and/or poorly ranked outcomes. CIs (95\%) were calculated for each importance score using bootstrapping. Importance scores were used to identify the top 10 research priorities for all respondents, people with PF, caregivers and $\mathrm{HCPs} /$ researchers, respectively.

All calculations were performed using Microsoft Excel 2013 or Stata/MP (V.15.1; StataCorp, Texas, USA).

\section{RESULTS}

Stage 1

One hundred and ninety-six questionnaires were completed in stage 1. Characteristics of respondents are described in table 1
Table 1 Characteristics of respondents of the identifying priorities and prioritisation questionnaires

\begin{tabular}{|c|c|c|}
\hline Characteristic & $\begin{array}{l}\text { Stage } 1 \text { Identifying } \\
\text { priorities questionnaire } \\
(\mathrm{n}=196)\end{array}$ & $\begin{array}{l}\text { Stage } 3 \\
\text { Prioritisation } \\
\text { questionnaire } \\
(\mathrm{n}=261)\end{array}$ \\
\hline \multicolumn{3}{|l|}{ Category of respondent } \\
\hline Person with PF & $152(78)$ & $134(51)$ \\
\hline Caregiver of person with PF & $32(16)$ & $38(15)$ \\
\hline $\begin{array}{l}\text { Healthcare professionals/ } \\
\text { researchers }\end{array}$ & $12(6)$ & $89(34)$ \\
\hline \multicolumn{3}{|l|}{ Gender } \\
\hline Male & $91(46)$ & $124(48)$ \\
\hline Female & $98(50)$ & $110(42)$ \\
\hline Not reported & $7(4)$ & $27(10)$ \\
\hline $\begin{array}{l}\text { Current age, years (people with } \\
\text { PF and caregivers) }\end{array}$ & $(n=184)$ & $(n=172)$ \\
\hline$\leq 54$ & $9(5)$ & $10(6)$ \\
\hline $55-64$ & $19(10)$ & $12(7)$ \\
\hline $65-74$ & $82(45)$ & $74(43)$ \\
\hline $75-84$ & $61(33)$ & $55(32)$ \\
\hline$\geq 85$ & $10(5)$ & $12(7)$ \\
\hline Not reported & $3(2)$ & $9(5)$ \\
\hline $\begin{array}{l}\text { Residence (people with PF and } \\
\text { caregivers) }\end{array}$ & $(n=184)$ & $(n=172)$ \\
\hline ACT & $3(2)$ & $3(2)$ \\
\hline NSW & $60(33)$ & $71(41)$ \\
\hline NT & $3(2)$ & $2(1)$ \\
\hline QLD & $17(9)$ & $13(8)$ \\
\hline SA & $19(10)$ & $14(8)$ \\
\hline TAS & $11(6)$ & $7(4)$ \\
\hline VIC & $56(30)$ & $45(26)$ \\
\hline WA & $12(7)$ & $8(4)$ \\
\hline Not reported & $0(0)$ & $9(5)$ \\
\hline $\begin{array}{l}\text { Years since diagnosed (people } \\
\text { with PF) }\end{array}$ & $(n=152)$ & $(n=134)$ \\
\hline $0-1$ & $12(8)$ & $16(12)$ \\
\hline $2-4$ & $71(47)$ & $115(86)$ \\
\hline$\geq 5$ & $65(43)$ & $59(44)$ \\
\hline Don't know & $1(1)$ & $3(2)$ \\
\hline $\begin{array}{l}\text { Relation of caregiver to person } \\
\text { with PF (caregivers) }\end{array}$ & $(n=32)$ & $(n=38)$ \\
\hline Husband & $12(37)$ & $11(29)$ \\
\hline Wife & $14(44)$ & $15(40)$ \\
\hline Partner & $0(0)$ & $1(2)$ \\
\hline Child & $5(16)$ & $5(13)$ \\
\hline Other & $1(3)$ & $0(0)$ \\
\hline Not reported & $0(0)$ & $6(16)$ \\
\hline $\begin{array}{l}\text { Health } \\
\text { professionalspeciality(>1option } \\
\text { allowed) }\end{array}$ & $(n=12)$ & $(n=89)$ \\
\hline Allied health professional & $3(25)$ & $15(17)$ \\
\hline Nurse & $1(8)$ & $6(7)$ \\
\hline
\end{tabular}




\begin{tabular}{lll}
\hline Table 1 Continued & & \\
\hline & $\begin{array}{l}\text { Stage 1 Identifying } \\
\text { priorities questionnaire } \\
(\mathbf{n}=196)\end{array}$ & $\begin{array}{l}\text { Stage 3 } \\
\text { Prioritisation } \\
\text { questionnaire } \\
(\mathbf{n}=261)\end{array}$ \\
\hline Characteristic & $1(8)$ & $10(11)$ \\
\hline Researcher & $0(0)$ & $38(43)$ \\
\hline Respiratory physician & $7(58)$ & $12(13)$ \\
\hline Specialist ILD physician & $0(0)$ & $2(2)$ \\
\hline Physicianandresearcher & $0(0)$ & $12(13)^{*}$ \\
\hline Other & & $(\mathbf{n}=89)$ \\
\hline Years of experience working in & & $35(39)$ \\
PF (HCPs) & NA & $15(17)$ \\
\hline $0-5$ & NA & $15(17)$ \\
\hline $6-10$ & NA & $9(10)$ \\
\hline $11-15$ & NA & $15(17)$ \\
\hline $16-20$ & NA & \\
\hline $21+$ & &
\end{tabular}

Results are expressed in terms of numbers (\% of $n$ )

$\mathrm{NA}=$ not asked

*Others include: exercise physiologist $(n=1)$, respiratory attendant/trainee $(n=5)$, registrar $(n=3)$, physician trainee $(n=2)$, clinical psychologist $(n=1)$

ACT, Australian Capital Territory; HCPs, healthcare professionals; ILD, interstitial lung disease; NA, not asked; NSW, New South Wales; NT, Northern Territory; PF, pulmonary fibrosis; QLD, Queensland; SA, South Australia; TAS, Tasmania; VIC, Victoria; WA, Western Australia.

(column 2). In summary, $78 \%$ of all respondents were people with PF and $50 \%$ were women. Of respondents who were people with PF or caregivers, $45 \%$ were aged between 65 and 74 years with $44 \%$ of caregivers being wives of people with PF. Of the 152 with PF, 43\% had been diagnosed five or more years ago. We had representation from each state and territory in Australia, with the distribution of participants consistent with that in the AIPFR and the general Australian population. ${ }^{14}$
Of the 560 individual questions extracted from the questionnaires, thematic analysis identified 52 initial themes based on 'key words' that encompassed all 560 responses. For example, 'Quicker/better/more certain diagnosis - early detection, better prognosis' was based on the inclusion of key words such as 'early detection' and 'better prognosis'. In total, there were four questions that included such words for which 87 of the 196 respondents mentioned these words in the research questions they submitted. The themes were then compared and discussed to generate 14 overarching themes to discuss at the stage 2 workshop. (table 2) (online supplemental file 2).

\section{Stage 2}

The stage 2 face-to-face workshop involved a total of 32 participants who were randomly allocated to one of five small groups. Of the 32 participants, $63 \%(n=20)$ were people with PF, $15 \%(n=5)$ were caregivers and $22 \%(n=7)$ were HCPs and researchers. A total of 53 specific research questions were generated from small group discussions. A review of questions by three researchers (GT, AEH and AT) identified 14 overlapping questions. For example, two questions read as 'What breathing techniques improve life participation?' and 'Breathing retraining/ strategies (trumpet playing!)'. These were incorporated into the question 'Can breathing exercises improve symptoms and life participation in people with PF?' For the majority of questions, the wording was not rephrased by the researchers but rather they are reported verbatim as expressed by participants during the workshop. This resulted in a final list of 39 research questions for prioritisation. (online supplemental file 3 )

\section{Stage 3}

A total of 270 individuals completed the prioritisation questionnaire. However, $9(3 \%)$ were incorrectly completed leaving a total of $261(97 \%)$ valid questionnaires that form the basis of these analyses. Table 1 (column 3) describes the characteristics of the 261 respondents. Of these, 51\% were people with PF,

Table 2 Stage 1 - overarching themes identified from the responses to the stage 1 questionnaire $(n=560)$

\begin{tabular}{|c|c|c|}
\hline Overarching themes & $\begin{array}{l}\text { Number of questions } \\
\text { incorporated into } \\
\text { final theme }\end{array}$ & $\begin{array}{l}\% \text { of respondents } \\
\text { for final themes } \\
(n=196)\end{array}$ \\
\hline (1) Quicker/better/more certain diagnosis - early detection, better prognosis & 4 & $44 \%$ \\
\hline $\begin{array}{l}\text { (2) Better treatments - fewer side effects, reduce disease progression, prolong life, lung transplant eligibility and post- } \\
\text { transplant medications }\end{array}$ & 6 & $40 \%$ \\
\hline (3) Understand the cause of PF - family history, occupation, exposures, mechanism & 4 & $39 \%$ \\
\hline (4) Improve education / information / awareness - reduce stigma, understand experience of living with PF & 5 & $35 \%$ \\
\hline (5) Relieve symptoms - breathlessness, cough, fatigue & 5 & $29 \%$ \\
\hline (6) Find a cure for PF - provide hope for those with disease & 1 & $24 \%$ \\
\hline (7) Better supportive care - optimal exercise and rehab programmes, oxygen therapy, diet, end of life planning and care & 6 & $19 \%$ \\
\hline (8) Psychosocial support for patients and carers-awareness of support groups and where to seek assistance & 6 & $16 \%$ \\
\hline (9) Improve self-management - empower individual to deal with disease & 6 & $12 \%$ \\
\hline (10) Improve quality of life for patients and carers - ability to perform basic tasks, dependency on carers & 2 & $8 \%$ \\
\hline (11) Access to research and clinical trials - access to trials, information on research & 3 & $8 \%$ \\
\hline $\begin{array}{l}\text { (12) Better access to care, especially in rural/regional areas - need to travel long distances for care, impact on patient and } \\
\text { carers }\end{array}$ & 2 & $6 \%$ \\
\hline (13) Understand my prognosis - stages of progression, life expectancy, what to expect & 2 & $3 \%$ \\
\hline (14) Decrease costs of care - long-term use of oxygen expensive & 1 & $1 \%$ \\
\hline
\end{tabular}




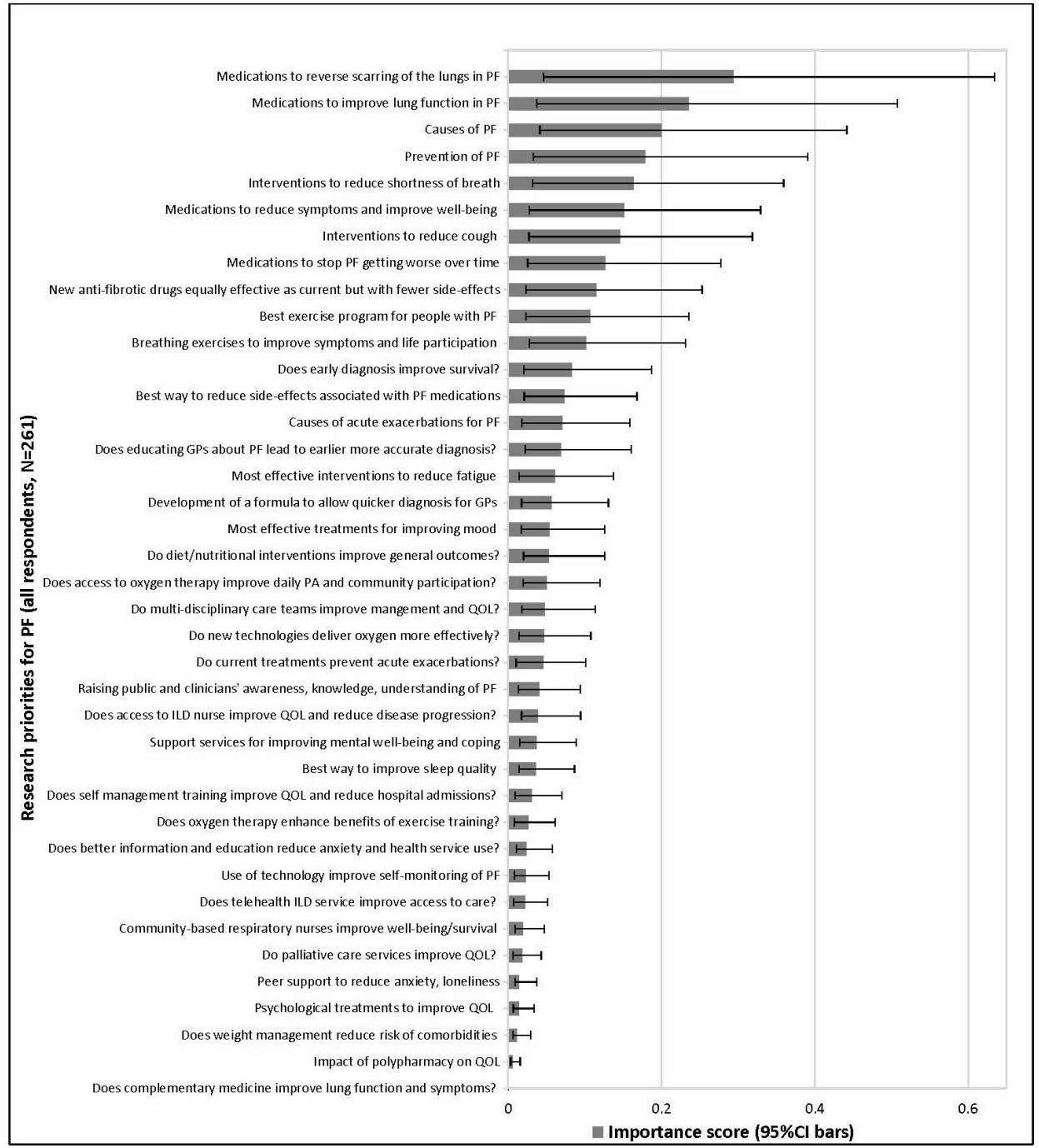

Figure 1 Overall importance scores based on all stakeholders ( $95 \% \mathrm{CI}$ bars). GPs, general practitioners; ILD, interstitial lung disease; PA, physical activity; PF, pulmonary fibrosis; QOL, qualityof life.

$34 \% \mathrm{HCP} /$ researchers and 15\% were caregivers (15\%) with $48 \%$ of respondents being men. Of the respondents who were people with PF or caregivers, 43\% were aged between 65 and 74 years, with $40 \%$ of caregivers being wives of people with $\mathrm{PF}$, and $44 \%$ of people with PF having been diagnosed five or more years ago. For HCPs/ researchers, $41 \%$ of the 89 respondents reported their speciality as being respiratory physician and within this group, $39 \%$ had between 0 to 5 years of experience working in PF.

\section{Top 10 research priorities for PF}

Figure 1 shows the ranking and importance scores for each of the 39 research questions developed for prioritisation based on all participants. Table 3 (overall rank P1-P10) lists the final top 10 research priorities for PF. Four of the 10 priorities related to the development of medications (P1, P2, P6 and P8), two related to interventions aimed at alleviating symptoms (P5 and P8), two related to understanding the cause and prevention of PF (P3 and $\mathrm{P} 4$ ) and one on the best exercise programme for people with PF (P10).

The rankings for each of the stakeholder groups respectively are provided in online supplemental file 4 (4A - people with $\mathrm{PF}$, 4B - caregivers, 4C - HCPs/researchers) while table 3 provides the respective top 10 priorities. Comparing across stakeholder groups, there was generally good consensus between the groups on the top 10 priorities, however, the actual rank varied. Caregivers and HCPs/researchers identified some research priorities that were not included in the overall top 10 list. For example, caregivers ranked 'Does educating GPs about PF lead to an earlier or more accurate diagnosis of PF?' as their ninth most important priority, while HCPs/researchers prioritised 'What are the causes of acute exacerbations?’ as their eighth top priority. (table 3 ).

\section{DISCUSSION}

This is the first research priority setting exercise undertaken specifically for PF that involved people with PF, caregivers, HCPs and researchers. We have identified the top 10 list of research questions for PF determined by stakeholders as a collective. These covered three broad areas: the development of new pharmacological medications aimed at preserving lung function, interventions to help manage chronic symptoms and identifying the causes of PF.

Overall, there was good consensus across stakeholders in terms of what questions were included in their top 10 research priorities. That is, people with PF nominated the same eight questions in their top 10 list as caregivers and HCPs/researchers. 


\begin{tabular}{|c|c|c|c|c|}
\hline $\begin{array}{l}\text { Overall rank } \\
(\mathrm{n}=261)\end{array}$ & $\begin{array}{l}\text { Research question } \\
\text { (importance score) }\end{array}$ & $\begin{array}{l}\text { People with PF } \\
\text { ( } n=134) \\
\text { Rank (IS) }\end{array}$ & $\begin{array}{l}\text { Caregivers } \\
(\mathrm{n}=38) \\
\text { Rank (IS) }\end{array}$ & $\begin{array}{l}\text { HCPs/researchers } \\
(\mathrm{n}=89) \\
\text { Rank (IS) }\end{array}$ \\
\hline P1 & What medications can reverse scarring in the lungs of people with PF? (0.294) & $1(0.300)$ & $1(0.359)$ & $1(0.256)$ \\
\hline P2 & What medications can improve lung function in people with PF? (0.235) & $2(0.252)$ & $2(0.271)$ & $5(0.195)$ \\
\hline P3 & What are the causes of PF? $(0.200)$ & $3(0.188)$ & $3(0.198)$ & $3(0.219)$ \\
\hline P4 & How can we prevent PF? (0.179) & $6(0.151)$ & $5(0.162)$ & $2(0.229)$ \\
\hline P5 & $\begin{array}{l}\text { What are the most effective interventions to reduce shortness of breath in people with PF? } \\
(0.164)\end{array}$ & $4(0.161)$ & $10(0.108)$ & $6(0.190)$ \\
\hline P6 & What medications can reduce symptoms and improve well-being in PF? (0.151) & $8(0.131)$ & $>10$ & $4(0.202)$ \\
\hline P7 & What are the most effective interventions to reduce cough in people with PF? $(0.146)$ & $5(0.153)$ & $8(0.123)$ & $7(0.144)$ \\
\hline P8 & What medications can stop PF getting worse over time? (0.126) & $9(0.125)$ & $6(0.161)$ & $9(0.113)$ \\
\hline P9 & $\begin{array}{l}\text { Can we develop anti-fibrotic drugs for PF that are equally effective as current options but } \\
\text { with fewer side effects? (0.115) }\end{array}$ & $7(0.137)$ & $7(0.128)$ & $>10$ \\
\hline P10 & What is the best exercise programme for people with PF? (0.106) & $10(0.117)$ & $>10$ & $>10$ \\
\hline$>10$ & Can breathing exercises improve symptoms and life participation in people with PF? & $>10$ & $4(0.166)$ & $>10$ \\
\hline$>10$ & Does educating GPs about PF lead to an earlier or more accurate diagnosis of PF? & $>10$ & $9(0.116)$ & $>10$ \\
\hline$>10$ & What are the causes of acute exacerbations of PF? & $>10$ & $>10$ & $8(0.117)$ \\
\hline$>10$ & Does early diagnosis improve survival for PF? & $>10$ & $>10$ & $10(0.109)$ \\
\hline
\end{tabular}

$>10$ indicates question was not ranked in the top 10.

GPs, general practitioners; HCPs, healthcare professionals; IS, important score; P, priority; PF, pulmonary fibrosis.

For people with PF and caregivers, their top three priorities focussed on finding medications for improving lung function and understanding the aetiology of PF. Given breathlessness and cough are the most debilitating and prevalent of unmanaged PF symptoms, it is not surprising that these areas ranked as the most important. ${ }^{15}$ However, there was some discordance between patients/caregivers and $\mathrm{HCPs} /$ researchers in terms of the top 3 and top 10 priorities with $\mathrm{HCPs} /$ researchers ranking prevention of $\mathrm{PF}$ as their second priority. In addition, $\mathrm{HCPs} /$ researchers included the importance of early diagnosis on survival and causes of acute exacerbations (AE) as top 10 priorities. This may be attributed to the different perspectives and experiences between people with PF and HCPs who deal with the management of the disease. Given an AE-IPF is associated with a mortality rate as high as $85 \%$ and mean survival periods of between 3 to 13 days, it is understandable that this challenging area would rate highly in the priority list for HCPs. ${ }^{16}$

To date, there has been little previous work of this kind undertaken for PF. Our results showed that top priorities for stakeholders reflect many unmet supportive care needs of people with PF and caregivers as previously reported in qualitative studies and in a recent systematic review. Identified needs include effective treatments to address debilitating symptoms such as dyspnoea and cough, and medications that can reverse or halt the disease process. ${ }^{6}$ Of interest was the finding that research into the causes and prevention of PF were ranked as top 10 research priorities for people with $\mathrm{PF}$ and $\mathrm{HCPs} /$ researchers. Loss of independence, becoming a burden on the family and fears of passing on the disease to other family members are reported in the literature to be prominent concerns for people with PF and caregivers, providing possible insight into why cause and prevention research questions were considered such high priorities. ${ }^{17}$ This finding also demonstrates how the impact of living with the disease and the challenges associated with its treatment and management are critical areas requiring research attention and highlights the role that discovery research will play in addressing such priorities.

Growing evidence indicates that non-pharmacological therapies such as pulmonary rehabilitation have important effects on symptoms, functional capacity and well-being in people with $\mathrm{PF}^{18}$ The inclusion of an optimal exercise programme for $\mathrm{PF}$ as a top 10 priority suggests that such interventions continue to play an important role in the management of PF and warrant further consideration.

Several prevalent themes from stage 1 , focussed on rapid and accurate diagnosis, improving education/awareness of the disease, better psychosocial support for patients and carers and self-management, were not ranked in the final top 10 in stage 3. This may stem from the proportion of stakeholders who participated in stages 1 and 3. In stage 1 , the ratio of $\mathrm{HCPs} /$ researchers to people with PF was 1:13 however, this ratio was more balanced at 1:1.5 in the prioritisation stage. Therefore, we feel that a more indicative representation of stakeholders' priorities emerged from the final prioritisation.

The list of research questions ranked as the top 10 priorities are not prescriptive, rather they will allow researchers to develop more specific hypotheses to test in these priority areas. To achieve this end will require experts, researchers and stakeholders to work collaboratively to identify and develop research proposals based on specific, answerable research questions. Consensus on proposals should consider: relevance (burden of disease, equity and evidence gaps), appropriateness (scientific rigour and suitability of methodology to answer research question), significance of research outcomes (impact, innovation and capacity building), feasibility (team quality and research environment) and value for money (potentially cost effective). ${ }^{19}$ In order to inform future work, an evaluation of the process should also be undertaken, to understand the impact of the research priority setting process. This would require outcome measures of performance that can be monitored and evaluated over time, 
evaluating process indicators such as stakeholder satisfaction, transparency of process and successful submission of research proposals.

The strength of this study is that all stakeholder groups across Australia were involved in identifying the top 10 research priorities for PF and the process was conducted using a systematic method. Limitations include stakeholders being from Australia, English speaking and not having data on ethnicity. In addition, our recruitment of HCPs did not specifically target any specific group such as palliative care providers who may have made an important contribution to our findings. Limited resources prevented a comprehensive, systematic review of the literature prior to commencing the priority setting process, however the limited number of current treatments for PF makes it less likely that this would have limited the scope of the priority setting exercise. We modified the JLA process to meet the needs of our patient group as reported in previous studies by holding a smaller face-to-face meeting in stage 2, followed by an online survey for stage $3 .{ }^{20}$ This decision was made due to the challenges of travelling to a face-to-face meeting for many people with PF, particularly in Australia where travel distances are considerable, and our desire to maximise the engagement of the PF community in stage 3 using online methods. While this enabled a broader participation in the choice of the final priorities, it could have affected the outcome.

\section{CONCLUSION}

This research priority setting exercise for PF has involved all stakeholders in identifying the top 10 research priorities for PF. Research aimed at PF prevention, development of interventions to improve lung function and alleviating symptom burden were identified as being most important to stakeholders. These findings will help guide future research directions.

\section{Author affiliations \\ ${ }^{1}$ Department of Allergy, Immunology and Respiratory Medicine, Monash University, Melbourne, Victoria, Australia \\ 2Department of Physiotherapy, La Trobe University, Melbourne, Victoria, Australia ${ }^{3}$ National Health and Medical Research Council Centre of Research Excellence in Pulmonary Fibrosis, The University of Sydney, Sydney, New South Wales, Australia ${ }^{4}$ Sydney School of Public Health, The University of Sydney, Sydney, New South Wales, Australia \\ ${ }^{5}$ Department of Respiratory Medicine, Royal Prince Alfred Hospital, Camperdown, New South Wales, Australia \\ ${ }^{6}$ School of Medicine (Central Clinical School), The University of Sydney, Sydney, New South Wales, Australia \\ ${ }_{7}^{7}$ Lung Foundation Australia, Milton, Queensland, Australia \\ ${ }^{8}$ Department of Respiratory and Sleep Medicine, Alfred Health, Melbourne, Victoria, Australia \\ ${ }^{9}$ Institute of Respiratory Health, Perth, Western Australia, Australia \\ ${ }^{10}$ Department of Physiotherapy, Alfred Health, Melbourne, Victoria, Australia}

\section{Twitter Allison Tong @allisontong1}

Acknowledgements The study acknowledges the time and contribution of all participants. With permission, we acknowledge by name the people with pulmonary fibrosis and caregivers who were able to participate in the workshop: Christine Cappello, Brian Conners, Gordian D'Souza, Tony Hyams, Jillian Block, Marilyn Bradford, Allan Buck, Leigh Buesnel, Kathleen Constantine, Brian Constantine, Patricia Crockford, Pam Davies, Greg Davies, Rob Graham, Hazel Ireson, Eli Light, Stuart McMillan, Jennifer Moran, Carole Musticone, John Price, Barbara Rowan, Bill Van Nierop, Theresia Van vugt, Beb Webb. In addition, we acknowledge Karen Symons for her support and assistance in recruiting participants and running the workshop.

Contributors Conceptualisation: GT, AT, AEH, TJC. Data curation: GT, AT, JYTL, AEH.
Formal analysis: GT, AEH, AT. Methodology: GT, AEH, AT. Writing—original draft: GT, AEH. Writing - review and editing: GT, AT, JYTL, TJC, AJHC, MB, TC, ING, JP, JM, AEH.

Funding This work was funded by the NHMRC Centre of Research Excellence in Pulmonary Fibrosis (GNT1116371) and supported by the Australian IPF Registry, Lung Foundation Australia and industry partners including Boehringer Ingelheim, Roche and Galapagos.

Competing interests None declared.

Patient consent for publication Not required.

Ethics approval The study protocol was approved by the La Trobe Human Ethics Committee (\#HEC19215) and the Sydney Local Health District Human Ethics Committee (2019/ETH111649).

Provenance and peer review Not commissioned; externally peer reviewed.

Data availability statement Data sharing not applicable as no data sets generated and/or analysed for this study. Data is qualitative rather than quantitative and its use has been approved for this study only.

\section{REFERENCES}

1 Behr J, Kreuter M, Hoeper MM, et al. Management of patients with idiopathic pulmonary fibrosis in clinical practice: the INSIGHTS-IPF registry. Eur Respir J 2015:46:186-96

2 Flaherty KR, Wells AU, Cottin V, et al. Nintedanib in progressive fibrosing interstitial lung diseases. N Engl J Med 2019;381:1718-27.

3 Crowe S, Fenton M, Hall M, et al. Patients', clinicians' and the research communities' priorities for treatment research: there is an important mismatch. Res Involv Engagem 2015;1:2.

4 Lindell KO, Kavalieratos D, Gibson KF, et al. The palliative care needs of patients with idiopathic pulmonary fibrosis: a qualitative study of patients and family caregivers. Heart Lung 2017;46:24-9.

5 Rogliani P, Calzetta L, Cavalli F, et al. Pirfenidone, nintedanib and N-acetylcysteine for the treatment of idiopathic pulmonary fibrosis: a systematic review and meta-analysis. Pulm Pharmacol Ther 2016;40:95-103.

6 Lee JYT, Tikellis G, Corte TJ, et al. The supportive care needs of people living with pulmonary fibrosis and their caregivers: a systematic review. Eur Respir Rev 2020;29. doi:10.1183/16000617.0125-2019. [Epub ahead of print: 30 Jun 2020].

7 Jo HE, Prasad JD, Troy LK, et al. Diagnosis and management of idiopathic pulmonary fibrosis: thoracic Society of Australia and New Zealand and lung Foundation Australia position statements summary. Med J Aust 2018;208:82-8.

8 Holland AE, Fiore JF, Bell EC, et al. Dyspnoea and comorbidity contribute to anxiety and depression in interstitial lung disease. Respirology 2014;19:1215-21.

9 The James Lind Alliance. The James Lind Alliance Guidebook November 2018, 2018. Available: www.jla.nihr.ac.uk

10 Chalmers I, Atkinson P, Fenton M, et al. Tackling treatment uncertainties together: the evolution of the James Lind initiative, 2003-2013. J R Soc Med 2013;106:482-91.

11 Tong A, Synnot A, Crowe S, et al. Reporting guideline for priority setting of health research (Reprise). BMC Med Res Methodol 2019;19:243.

12 da Costa Santos CM, de Mattos Pimenta CA, Nobre MRC. The PICO strategy for the research question construction and evidence search. Rev Lat Am Enfermagem 2007; 15:508-11.

13 McMillan SS, King M, Tully MP. How to use the nominal group and Delphi techniques. Int J Clin Pharm 2016;38:655-62.

14 HE J, Glaspole I, Grainge C, et al. Baseline characteristics of idiopathic pulmonary fibrosis: analysis from the Australian idiopathic pulmonary fibrosis registry. Eur Respir J 2017:49.

15 Carvajalino S, Reigada C, Johnson MJ, et al. Symptom prevalence of patients with fibrotic interstitial lung disease: a systematic literature review. BMC Pulm Med 2018; 18:78.

16 Juarez MM, Chan AL, Norris AG, et al. Acute exacerbation of idiopathic pulmonary fibrosis-a review of current and novel pharmacotherapies. J Thorac Dis 2015;7:499-519.

17 Bridges JFP, Paly VF, Barker E, et al. Identifying the benefits and risks of emerging treatments for idiopathic pulmonary fibrosis: a qualitative study. Patient 2015;8:85-92.

18 Dowman L, Hill CJ, Holland AE. Pulmonary rehabilitation for interstitial lung disease. Cochrane Database Syst Rev 2014:Cd006322.

19 Australian Clinical Trials Alliance. Research prioritisation framework, 2020. Available: https://clinicaltrialsalliance.org.au/resource/acta-research-prioritisation-framework/

20 Rowbotham NJ, Smith SJ, Elliott ZC, et al. Adapting the James Lind alliance priority setting process to better support patient participation: an example from cystic fibrosis. Res Involv Engagem 2019;5:24. 